TITLE:

\title{
Emergence of multiple tori structures in a single polyelectrolyte chain
}

\author{
AUTHOR(S): \\ Sakaue, $T$
}

CITATION:

Sakaue, T. Emergence of multiple tori structures in a single

polyelectrolyte chain. JOURNAL OF CHEMICAL PHYSICS 2004, 120(13):

6299-6305

ISSUE DATE:

2004-04-01

URL:

http://hdl.handle.net/2433/49892

\section{RIGHT:}

Copyright 2004 American Institute of Physics. This article may be downloaded for personal use only. Any other use requires prior permission of the author and the American Institute of Physics. 


\title{
Emergence of multiple tori structures in a single polyelectrolyte chain
}

\author{
Takahiro Sakaue ${ }^{\text {a) }}$ \\ Department of Physics, Graduate School of Science, Kyoto University and CREST, Kyoto 606-8504, Japan
}

(Received 1 December 2003; accepted 9 January 2004)

\begin{abstract}
We investigated the collapsed structure of a weakly charged wormlike chain under a moderate concentration of 1:1 electrolyte solution. By assuming a torus as a grand state, we found that the size of a torus is determined by the balance between surface energy and electrostatic energy, which leads to a finite torus thickness almost independent of the chain contour length. Owing to this unique characteristic, a long charged wormlike chain forms multiple tori structure as a collapsed product, which is never seen with a neutral wormlike chain. These features were confirmed by a Monte Carlo simulation. (c) 2004 American Institute of Physics. [DOI: 10.1063/1.1652433]
\end{abstract}

\section{INTRODUCTION}

When a polymer chain is immersed in a poor solvent, its conformation changes from a statistical coil into a compact collapsed state. This conformational transition, so-called coil-globule transition, has been the subject of extensive studies in polymer physics. $^{1-3}$ The nature of this coilglobule transition critically depends on the chain stiffness. ${ }^{2-4}$ In the case of flexible chains, a gaslike coil gradually shrinks as the solvent quality becomes worse, and a liquidlike globule is formed in poor solvent, which is characterized as a disorder-disorder transition. On the other hand, an abrupt first-orderlike transition is expected for stiff or semiflexible chains, suggesting the possible occurrence of a disorderorder transition. Actually, it is known that a chain with uniform rigidity (wormlike chain) forms a torus by wrapping upon itself circumferentially. ${ }^{5-11}$ In fact, many of the conformational properties of DNA molecules can be modeled quite well by a wormlike chain, and electron microscopy has revealed that the most common morphology of collapsed DNA is a torus. ${ }^{12-15}$ Computer simulation studies have also shown that, for a stiff chain with wormlike flexibility, a torus shape is the most stable over a wide range of conditions. ${ }^{15-21}$

If a polymer chain bears ionizable groups, i.e., polyelectrolyte, long-range electrostatic interaction acts between charged groups. This has a significant influence on the overall shape of the collapsed polymer chain in a poor solvent as well as the nature of the coil-globule transition. In the case of a flexible polyelectrolyte, a single globule may become unstable due to the balance between the surface energy and the electrostatic energy. Scaling theory predicts that the resulting structure consists of multiple small collapsed globules connected by strings, or a so-called pearl-necklace. ${ }^{22}$ This pearl-necklace structure has been further supported by a variational approach ${ }^{23}$ and intense computer simulations. ${ }^{24-26}$ Such pearl-necklace structures are regarded as micro-segregation with liquidlike mini-globules. On the contrary, it has recently been shown that multiple tori structure is generated from single giant DNA molecules by the addition of certain cationic surfactants. ${ }^{27}$

a)Electronic mail: sakaue@chem.scphys.kyoto-u.ac.jp
Although, as described above, both the stiffness and Coulomb interaction have a great significance on the collapsed structure of a polymer chain, there seems to have been almost no studies on how these two factors work simultaneously. In particular, synergy between these two factors is expected to be important for the collapsing behavior of DNA molecules. In fact, there is an early report on the possible role of electrostatic interaction in the striking feature of DNA condensation that the size distribution of multi-molecular DNA condensates is independent of the DNA molecular length. $^{13}$

In the present study, we investigate the structure of a single semiflexible chain with uniform rigidity and charge density (uniformly charged wormlike chain) in a poor solvent. We consider the case with high screening, i.e., the solvent contains a moderate concentration of 1:1 salt. Thus, the electrostatic interaction is screened at the monomer scale. Assuming a torus as a collapsed structure, we discuss how its size depends on the chain length and other physical parameters, and demonstrate qualitative differences from a neutral wormlike chain. We also point out the possible formation of multiple tori, which is improbable for a neutral wormlike chain. These predictions are discussed in comparison with the results of a Monte Carlo simulation.

\section{THEORY}

We consider an infinitely dilute polyelectrolyte solution with wormlike flexibility in the presence of 1:1 electrolyte with concentration $c$. A polyelectrolyte chain is characterized by the total contour length $L$, the length of Kuhn segment $l$, its thickness $d$, and the linear charge density $\rho$. In our notation, $l$ is solely attributed to the nonelectrostatic mechanical rigidity. There are two characteristic lengths associated with electrostatic interactions, the Bjerrum length $l_{B}=e^{2} / \epsilon k_{B} T$ and the Debye length $r_{D}=\left(8 \pi l_{B} c\right)^{-1 / 2}$, where $e, \epsilon$, and $k_{B} T$ denote the unit charge, the dielectric constant of the solvent and thermal energy, respectively. We consider the case where $\rho l_{B}<1$. Thus, the effects of salt ions are simply reduced to conventional linear screening with a decay length of $r_{D}$. In a poor solvent, a chain is collapsed into a compact structure. For the global shape of the collapsed structure of a charged 
wormlike chain, we first assume a single torus, by analogy with a neutral wormlike chain. The torus is characterized by two radii of curvature: The average radius $R$, and the thickness $r$ of the torus. We also assume that the torus is composed of densely packed stiff segments in a parallel alignment, which leads to the relationship $\pi d^{2} L / 4=2 \pi^{2} r^{2} R$.

To discuss the optimum shape of the torus, we adopt the following semigrand potential (the ensemble considered is specified by the fixed chain length and the fixed chemical potential of the $1: 1$ salt)

$$
\Omega=\Omega_{\text {attr }}+\Omega_{\text {surf }}+\Omega_{\text {bend }}+\Omega_{\text {elec }}+\Omega_{\text {trans }}-\mu n \times 2 \pi R .
$$

The torus structure is stabilized by the attractive interaction between segments due to the poor solubility of the chain backbone, which produces the first two terms. For simplicity, we consider the case of a maximally dense torus. In this case, the volume energetic term $\Omega_{\text {attr }}$ does not depend on the torus shape. The second term is the surface energy

$$
\Omega_{\text {surf }}=\gamma S,
$$

where $\gamma$ is the surface tension, and $S=4 \pi^{2} r R$ is the surface area of a torus. The third term is the bending energy required to form the torus, and can be approximated as

$$
\Omega_{\text {bend }} \sim \kappa \frac{L}{R^{2}},
$$

where $\kappa=k_{B} T l / 2$ is the bending modulus. The remaining terms represent the effects associated with electrostatic interactions and are discussed below.

Although the chain considered here is weakly charged, the electrostatic self-energy becomes substantially large by forming a torus. Then, the competition between electrostatic energy $\Omega_{\text {elect }}$ and the translational freedom of the oppositely charged salt ions may play some roles. ${ }^{28,29}$ In this article, we consider the case with nonpenetrating salt ions, i.e., the salt ions do not penetrate into the torus, but rather condense onto its surface. We incorporate this possibility as follows. The electrostatic energy of a torus is evaluated in two steps. First, we calculate the electrostatic energy of a cylinder with radius $r$ and length $2 \pi R$ (without the end effect). We then also consider the change in electrostatic energy due to bending of the cylinder. This treatment is allowed as long as the mean radius of the torus is much larger than the Debye length. The distribution of salt ions can be formally treated within the framework of the Poisson-Boltzmann theory as long as the effect of electrostatic correlations between salt ions is negligible, as in our case. According to the solution of the Poisson-Boltzmann equation, the profile of salt ions (with a negative charge) is characterized by a high density in the vicinity of a highly charged cylinder (with a positive charge) and a rapid decrease outside of this region. We approximate such a distribution using a so-called two-zone model, where the negatively charged salt ions are considered to be either condensed within close proximity to the cylinder surface, or completely free, and thus occupying the entire volume uniformly. ${ }^{28}$ We will regard the salt ions as condensed if they are located within some distance $\delta$ from the cylinder surface. By denoting the number of condensed salt ions on the cylinder surface per unit length $n$, the spatial distribution of charge density $\rho_{q}(x)$ (where $x$ is the radial distance from the cylinder axis) is expressed as

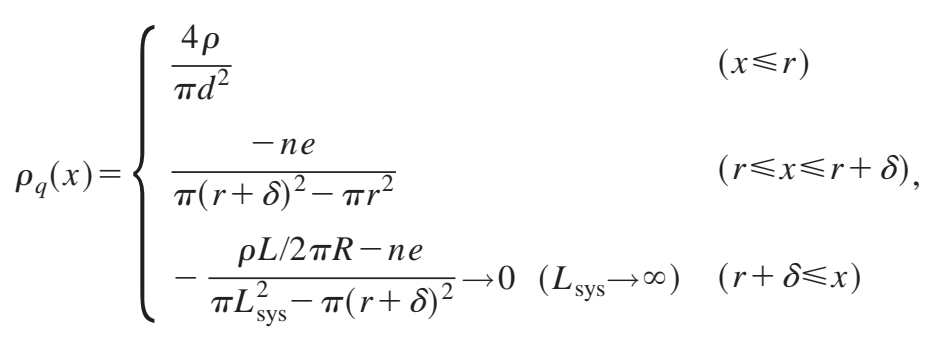

where $L_{\text {sys }}$ is the system size for a single chain, which becomes infinite at the limit of infinite dilution. With the help of axial symmetry and the Gauss theorem, the electric field $E\left(x_{0}\right)$ is calculated as

$$
E\left(x_{0}\right)=\frac{4 \pi}{x_{0} \epsilon} \int_{0}^{x_{0}} d x x \rho(x) .
$$

In reality, the dielectric constant inside the torus is different from that of the solvent. However, we do not consider this effect for the sake of simplicity. The electrostatic energy is obtained as

$$
\Omega_{\mathrm{elec}} \sim \frac{\epsilon \pi R}{2} \int_{0}^{r+r_{D}} d x\left[x E(x)^{2}\right]+\kappa_{e} \frac{2 \pi}{R},
$$

where $\kappa_{e}$ is the bending modulus of the cylinder due to electrostatic interaction. We approximate this modulus using the Odijk-Skolnick-Fixman theory with the renormalized charge density of the cylinder: ${ }^{30,31}$

$$
\begin{aligned}
\kappa_{e} & =k_{B} T l_{B} r_{D}^{2} \frac{\left[\int_{0}^{r+\delta} d x 2 \pi x \rho_{q}(x)\right]^{2}}{4}, \\
& =k_{B} T l_{B} r_{D}^{2} \frac{\left(4 \rho r^{2} / d^{2}-n e\right)^{2}}{4} .
\end{aligned}
$$

The fifth term in Eq. (1) represents the translational entropy of condensed salt ions

$$
\Omega_{\text {trans }}=2 \pi k_{B} \operatorname{Tn} R \ln \left\{\frac{n}{\pi(r+\delta)^{2}-\pi r^{2}}\right\} .
$$




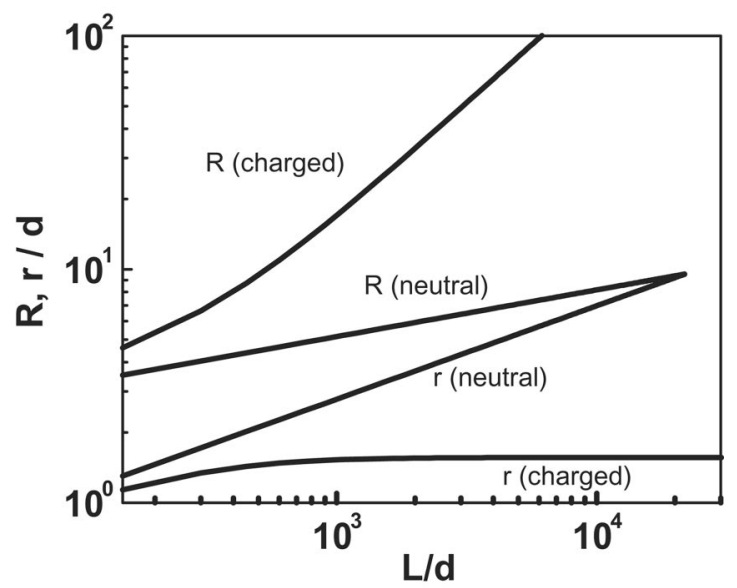

FIG. 1. Double-logarithmic plot of the torus size, average radius $R$, and thickness $r$ vs chain length $L$. The parameters adopted are as follows; $\gamma d^{2} / k_{B} T=4.0, l / d=15.0, \delta / d=0.15, r_{D} / d=1.0, l_{B} / d=0.2, \rho d=1.0$ (for a charged chain), and 0.0 (for a neutral chain).

The last term in Eq. (1) assures particle conservation, where $\mu=k_{B} T \ln c$ is the chemical potential of salt ions.

Using the relationship $\pi d^{2} L / 4=2 \pi^{2} r^{2} R$, which means that the segments inside the torus are most densely packed, the expression for the semigrand potential Eq. (1) is reduced to the two variables $r$ and $n$. By minimizing Eq. (1) with respect to these variables, one obtains the optimum salt ion distribution and the size of a torus. The corresponding semigrand potential with total contour length $L$ is denoted by $\Omega(L)$.

\section{RESULTS}

In Fig. 1, we show the dependence of the torus size $r, R$ on the chain contour length. For comparison, we also show results for a neutral chain $\left[l_{B}, \rho \rightarrow 0\right.$ in Eq. (1)]. As has been well established, the size of the most densely packed torus made of neutral wormlike chain can be described with the scaling $r \sim L^{2 / 5}, R \sim L^{1 / 5}$. In our model with $l_{B}, \rho \rightarrow 0$ in Eq. (1), these take the form

$$
r \sim\left(\frac{\gamma d^{6} L^{2}}{\kappa}\right)^{1 / 5}, \quad R \sim\left(\frac{\kappa^{2} L}{\gamma^{2} d^{2}}\right)^{1 / 5} .
$$

As the chain length increases, the thickness of torus $r$ increases more rapidly than the mean radius $R$, and thus a torus cannot be formed beyond the critical length $L^{*}$, obtained as $R\left(L^{*}\right)=r\left(L^{*}\right)$.

On the other hand, a charged wormlike chain shows a qualitatively different length dependence, which is characterized by saturation of the torus thickness $r$. This thickness is determined as a function of the surface tension $\gamma$, linear charge density of the chain $\rho$, Bjerrum length $l_{B}$ and Debye length $r_{D}$. In Fig. 2, we show the dependence of the thickness $r$ on these parameters. The thickness $r$ increases with an increase in $\gamma$ and decreases in $\rho, l_{B}$ and $r_{D}$ (or equivalently with an increase in the $1: 1$ salt concentration $c$ ).
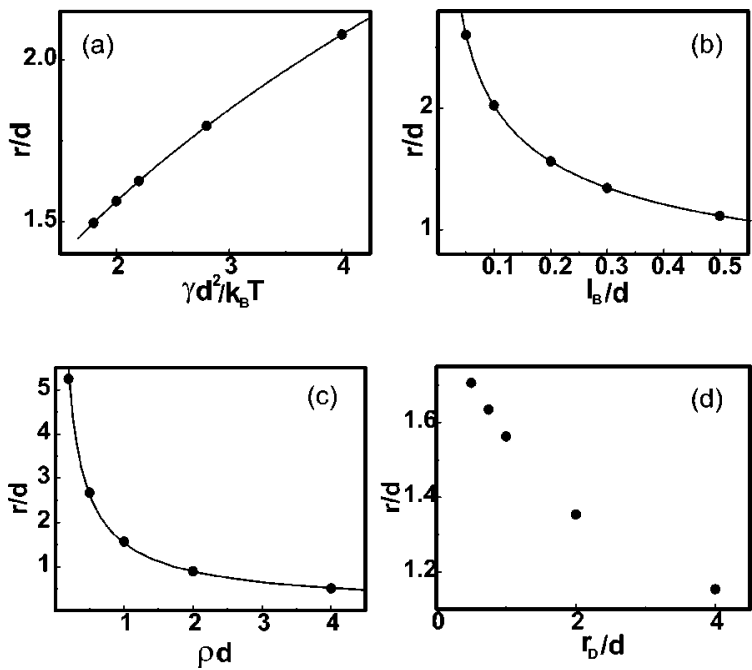

FIG. 2. Dependence of the torus thickness $r$ on the (a) surface tension $\gamma$, (b) Bjerrum length $l_{B}$, (c) linear charge density of a chain $\rho$ and (d) Debye length $r_{D}$. We define the thickness as the optimum value of $r$ for the chain length $L / d=6.0 \times 10^{4}$. The parameters are the same as those in Fig. 1. The lines in (a)-(c) are the fittings by a power function, with exponents of 0.41 , -0.37 , and -0.77 , respectively.

\section{DISCUSSION}

\section{A. What determines the torus size?}

A prominent structural property of a torus composed of charged polymers is its finite thickness $r$, i.e., $r$ is almost independent of the chain length $L$. The qualitative aspect of this phenomenon can be easily understood as follows. The finite nature of the torus thickness is a result of competition between the surface energy $\left[\Omega_{\text {surf }}\right.$ in Eq. (1)] and the electrostatic self-energy $\left[\Omega_{\text {elec }}\right.$ in Eq. (1)] of the torus. If we neglect the possibility of the condensation of salt ions on the torus surface and the effect of the electrostatic persistence length [second term in Eq. (6)], the electrostatic self-energy of a torus can be written as

$$
\Omega_{\mathrm{elec}} \sim k_{B} T l_{B} \frac{(\rho L)^{2}}{R}\left[\frac{1}{4}+\ln \left\{\frac{r+r_{D}}{r}\right\}\right] .
$$

By further neglecting the logarithmic dependence on $r$ in Eq. (11), it becomes possible to derive an approximate analytic expression for $r$ and $R$.

$$
r \sim\left(\frac{\gamma d^{4}}{k_{B} T l_{B} \rho^{2}}\right)^{1 / 3}, \quad R \sim\left(\frac{\left(k_{B} T l_{B}\right)^{2} \rho^{4}}{\gamma^{2} d^{2}}\right) L .
$$

This is the case for a relatively highly charged chain $\left[\gamma^{2} d^{2} \kappa^{3} \ll\left(k_{B} T l_{B}\right)^{5} \rho^{10} L^{6}\right]$. Of course, the analysis with a more sophisticated model (Sec. II) does not show any precise power dependence of $r$ on these parameters. Nevertheless, fitting with a power function gives exponents that are somewhat close to the above simple analysis, implying that the difference between these analyses is mostly qualitative, except for the dependence on the salt concentration. To capture the effect of the salt concentration, which is interesting from an experimental point of view, we need a model with salt ions (Sec. II). Our model qualitatively agrees with a recent experiment on the effect of the salt concentration. ${ }^{32}$ It should 
be mentioned that the strong (almost linear) dependence of $R$ on $L$ may be observed only in a narrow interval of $L$, since a torus may split into multiple tori in a long chain (Secs. IV B and V).

When the electrostatic effect becomes weak (the chain is less charged and/or the Bjerrum length becomes small), and the chain becomes stiff, the bending energy $\left[\Omega_{\text {bend }}\right.$ in Eq. (1)] becomes more substantial than the electrostatic energy. In this case, the thickness is mostly determined by the competition between the surface energy and bending energy. In the limit of weak electrostatic effect $\left[\gamma^{2} d^{2} \kappa^{3}\right.$ $\gg\left(k_{B} T l_{B}\right)^{5} \rho^{10} L^{6}$ ], we obtain the neutral chain scaling [Eq. (10)]. Between these two limiting cases, the dependence of the torus size on the chain length shows a crossover from neutral chain scaling [Eq. (10)] for small $L$ to charged chain scaling [Eq. (12)] for large $L$.

\section{B. Multiple tori formation}

So far, we have considered only a single torus. Thus, the mean radius of a torus formed with charged wormlike chain $R$ increases almost linearly with the chain length while other parameters are fixed. However, if $R$ becomes sufficiently large, it is expected that a single large torus may split into multiple small tori. In this subsection, we consider this issue from a thermodynamic point of view. If the structure is composed of $m$ small tori, there are $(m-1)$ connecting parts. In these parts, the local structure is expected to deviate from the ideal one, and can be regarded as a kind of defect. By denoting the increase in the semigrand potential due to the presence of such defects as $\Delta \Omega$, the semigrand partition function with $m$ tori $\Xi_{m}$ is written as

$$
\Xi_{m}=\sum_{\left\{L_{i}\right\}} \exp \left\{-\frac{\Omega\left(m,\left\{L_{i}\right\}\right)+(m-1) \Delta \Omega}{k_{B} T}\right\},
$$

where $\Omega\left(m,\left\{L_{i}\right\}\right)=\sum_{i=1}^{m} \Omega\left(L_{i}\right)$ ( $L_{i}$ is the chain length required to form the $i$ th torus). A chain with contour length $L$, Kuhn length $l$ is considered to be composed of $N=L / l$ statistically independent segments. Therefore, to perform this calculation, a chain is fractionalized by the bin width with Kuhn segment, and the summation is made under the constraint $\sum_{i=1}^{m} L_{i}=L$. The probability that the folded structure is composed of $m$ tori $P(m)$ is

$$
P(m)=\frac{\Xi_{m} \exp \left(\frac{\mu_{t} m}{k_{B} T}\right)}{\sum_{n=1}^{\infty} \Xi_{n} \exp \left(\frac{\mu_{t} n}{k_{B} T}\right)},
$$

where the chemical potential of the torus $\mu_{t}=0$, since there is no conservation of the number of tori. In Fig. 3, we show

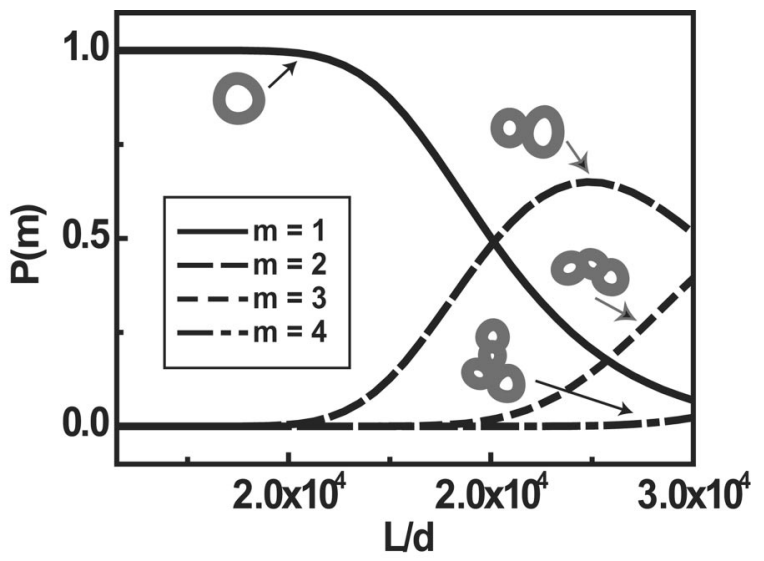

FIG. 3. Probabilities for the appearance of $m$ tori depending on the chain length. $\Delta \Omega / k_{B} T=1$, and other parameters are the same as those for Fig. 1.

the dependence of $P(m)$ on the chain contour length $L$. When the chain becomes longer, a single torus first splits into two tori. With a further increase in $L$, a structure with more tori becomes preferable.

These sequential structural changes can be explained by the property of the system that the torus thickness $r$ is limited to a finite value. Thus, the total volume and surface area of the collapsed structure do not depend on the number of tori $m$, if we ignore the connecting parts between neighboring tori. Therefore, the apparent contribution we should consider here is the bending energy $\left(\Omega_{\text {bend }}\right.$ ) and the increase in the semigrand potential due to the presence of connecting parts $(\Delta \Omega)$. While splitting a torus into multiple small tori requires penalties in both of these contributions, it still may occur due to entropic considerations. When the chain is long, and accordingly the mean radius of the torus is large, the bending energy cost due to splitting of the torus is rather small. On the other hand, the possible number of states significantly increases. In fact, the number of ways that $m$ tori can be formed from $N(=L / l)$ segments is $(N-1) ! /((N-m) !(m$ $-1)$ !), each of which has a different weight proportional to $\exp \left(-\Omega\left(m,\left\{L_{i}\right\}\right) / k_{B} T\right)$. The competition between these two tendencies leads to the sequential structural changes in a charged wormlike chain. We emphasize that such splitting of a torus is never expected with a neutral wormlike chain.

\section{MONTE CARLO SIMULATION}

An off-lattice Monte Carlo simulation has been performed to confirm the theoretical considerations discussed above. In the simulation, a chain is composed of $N$ spherical charged monomeric units with diameter $\sigma$ and valency $q$ connected by a harmonic bond potential with a cut-off:

$$
U_{\text {bond }}=\left\{\begin{array}{ll}
\kappa_{\text {bond }} \sum\left(\left|\boldsymbol{r}_{i}-\boldsymbol{r}_{i+1}\right|-\sigma\right)^{2} & \left(\text { if } l_{\min }<\left(\left|\boldsymbol{r}_{i}-\boldsymbol{r}_{i+1}\right|-\sigma\right)<l_{\max }\right) \\
\infty & \text { (otherwise) }
\end{array},\right.
$$




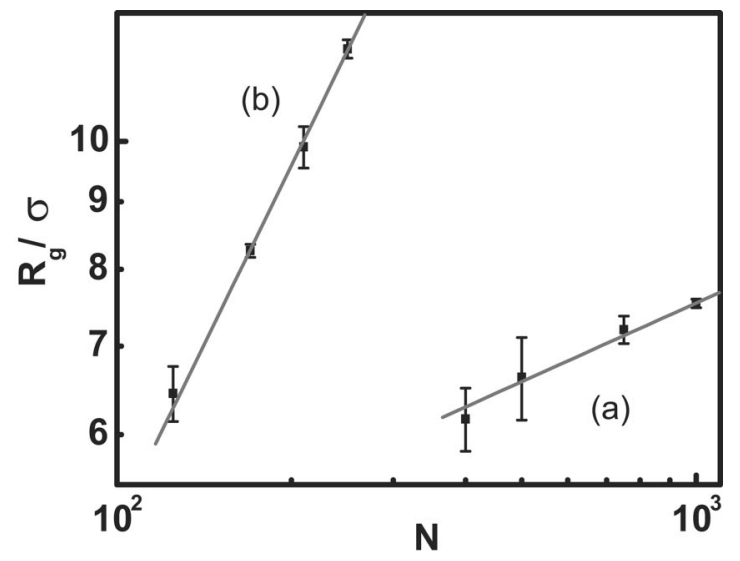

FIG. 4. Double-logarithmic plot of the gyration radius of (a) a neutral chain and (b) a charged chain vs chain length $N$ based on a Monte Carlo simulation. The slopes are (a) $0.197 \pm 0.019$ and (b) $0.897 \pm 0.022$, respectively.

where the spring constant $\kappa_{\text {bond }}=20 k_{B} T$, and $l_{\min }=0.85 \sigma$, $l_{\max }=1.15 \sigma$. To mimic a wormlike chain, stiffness is introduced by the following bending potential:

$$
U_{\text {bend }}=\sum \kappa_{\text {bend }}\left(1-\cos \left(\theta_{i}\right)\right)^{2},
$$

where $\theta_{i}$ is the angle between the $i$ th and $i+1$ th bonds. The bending rigidity parameter is set to be $\kappa_{\text {bend }}=30 k_{B} T$. The electrostatic interaction between monomeric units is implemented through the Debye-Huckel potential:

$$
U_{\mathrm{DH}}\left(r_{i, j}\right)=k_{B} T l_{B} \frac{q^{2}}{r_{i, j}} \exp \left(-\frac{r_{i, j}}{r_{D}}\right) .
$$

In addition, nonadjacent monomeric units interact through the truncated Lennard-Jones potential:

$$
U_{\mathrm{LJ}}\left(r_{i, j}\right)=\epsilon_{\mathrm{LJ}}\left[\left(\frac{\sigma}{r_{i, j}}\right)^{12}-\left(\frac{\sigma}{r_{i, j}}\right)^{6}-c\left(r_{c}\right)\right],
$$

where $\epsilon_{\mathrm{LJ}}$ controls the degree of poor solubility, and $c\left(r_{c}\right)$ $=\left(\sigma / r_{c}\right)^{12}-\left(\sigma / r_{c}\right)^{6}$ so that the potential value becomes zero at a cut-off distance of $r_{c}=2.5 \sigma$.

Simulations have been carried out for a charged chain $\left(q=1, l_{B} / \sigma=0.2, r_{D} / \sigma=1, \epsilon_{\mathrm{LJ}} / k_{B} T=1.8\right)$, and a neutral chain $\left(q=0, \epsilon_{\mathrm{LJ}} / k_{B} T=0.5\right)$.

The collapsed structure of a neutral chain with the present parameters is always a single torus for a chain lentgh of $N \in(400,1500)$. On the other hand, not only the torus but also rod structures as metastable states are formed in the case of a charged chain. These two morphologies can be distinguished by analyzing several structural measures. To avoid the mixing from the contribution of metastable rod structures, we monitored the following two indices: (1) The radial density profile of monomeric units around the center-of-mass of the chain, ${ }^{17}$ (2) the asphericity as a measure of the differences in spatial symmetry, which is calculated from the three eigenvalues of inertia of gyration tensor of the chain. ${ }^{16} \mathrm{We}$ also utilized the information from the snapshot, and only considered the torus structure in the following analysis.

In Fig. 4, we show how the gyration radius of the torus depends on the chain length. The gyration radius of the torus (a)

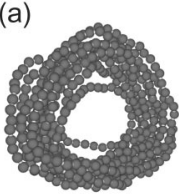

(b)
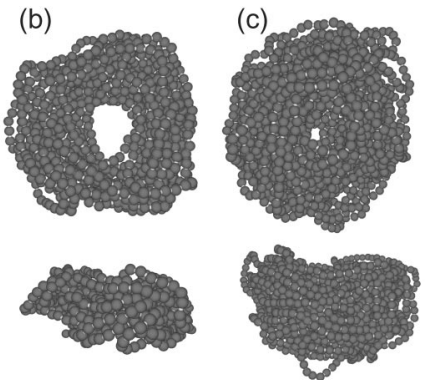

FIG. 5. Top and side views of collapsed neutral chains from Monte Carlo simulations. The chain length is (a) $N=500$, (b) $N=1000$, and (c) $N$ $=2000$.

for a charged chain rapidly increases with the chain length compared to that of a neutral chain. The scaling relation is

$$
R_{g} \sim N^{\nu}
$$

where $\nu=0.197 \pm 0.019$ for a neutral chain, and $\nu=0.897$ \pm 0.022 for a charged chain. These results correspond well with the theoretical argument in the present study, where $\nu$ $=0.2$, and $\nu \sim 1.0$, respectively. These features are clearly shown in snapshots from the simulation, as exemplified in Figs. 5 and 6. The torus of a neutral chain (Fig. 5) increases in thickness with an increase in the chain length. When the chain length is increased beyond the critical length, a neutral chain can not form a torus. At $N=2000$, we obtain a disklike structure rather than a torus [Fig. 5(c)]. In the case of a charged chain (Fig. 6), the torus becomes large with an increase in the chain length, while the thickness is almost constant, and is composed of three strands under the present conditions. However, with a further increase in the chain length, it is difficult for a long chain to form a large torus. In typical simulations with chain length $N=500$, two rings are

(a)

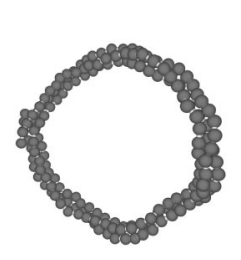

(b)

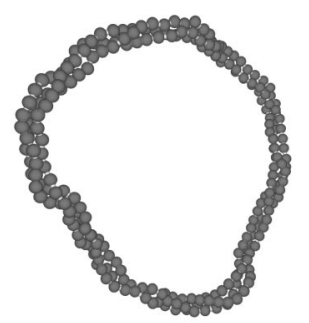

(c)

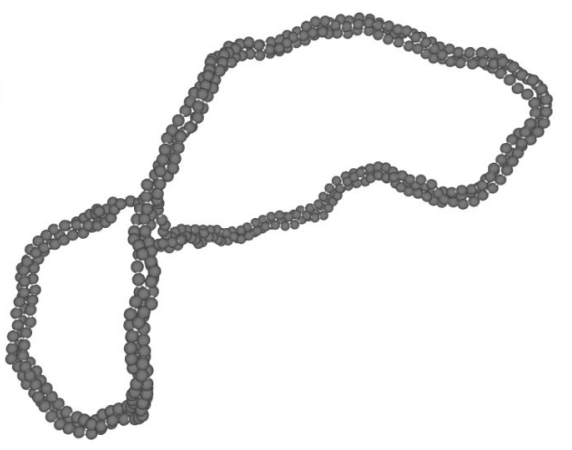

FIG. 6. Snapshots of collapsed charged chains from Monte Carlo simulations. The chain length is (a) $N=170$, (b) $N=250$, and (c) $N=500$. 
formed in different parts of the chain during thermal fluctuation. Both rings grow and a structure with two tori is finally obtained. Under the present conditions, it is practically impossible to relax into possible more stable states from this kinetically trapped state with a conventional canonical sampling. Thus, the multiple tori structure is formed due to kinetic effects, and we could not confirm our argument based on equilibrium theory in Sec. IV B.

Finally, we would like to mention an additional point on the torus structure for a charged chain. Interestingly, the strands wrap around each other to form a triple helix. Such helical structures formed of multiple strands of proteins are ubiquitously seen as basic architectures of living matters, such as collagen and actin. In our simulation, such a multistranded helix is spontaneously formed upon collapse without any specific chemical interaction such as hydrogen bonding. This seems to be a natural result, since the optimum packed structures of strings are helices. ${ }^{33}$ Our results indicate the possibility that the number of strands (thickness of the bundle) is controlled by adjusting apparent physical parameters.

\section{CONCLUSION}

We have studied the structure of a collapsed wormlike chain with a uniform linear charge density. We have specifically considered the case with weak electrostatic correlations and high screening, where the role of small electrolyte molecules in the system can almost be reduced to conventional linear screening. By assuming a torus as a collapsed structure, we have shown that the thickness of a torus made from a relatively highly charged chain is limited to a finite value. This thickness is determined by the balance between the surface energy and the electrostatic self-energy of the torus, and thus depends on physical parameters, such as surface tension, linear charge density of the chain, Bjerrum length and Debye length. We have also discussed the possibility of the formation of multiple tori for the collapsed structure of a single charged wormlike chain.

The dependence of the torus size on the chain length based on the results of a Monte Carlo simulation shows good agreement with the theory for both neutral and charged chains. In a long charged chain, the multiple tori is formed as a collapsed structure. In the present simulations, the multipe tori structure is generated due to the collapse kinetics rather than to equilibrium theory, as has been discussed in Sec. IV B. However, this does not mean that the kinetics are always the dominant factor, and it would be interesting to explore the situation in which the multiple tori structure is formed under equilibrium.

Finally, we would like to address the possible mapping of our theory into the case of DNA (and other strongly charged wormlike chain). DNA molecules are collapsed by the addition of various kinds of condensing agents, such as polyamines, neutral polymers, cationic surfactants, and alcohols. In this case, small positively charged ions in the solution renormalize the original DNA charge by counterion condensation, and also play important roles in generating attractive interaction. ${ }^{34,35}$ Despite the various physical origins of attractive interaction, it is possible to determine the structure of collapsed DNA with a renormalized charge under phenomenological attractive interaction between segments, i.e., with an effectively poor solvent for DNA molecules. If the interior charge of a DNA torus is completely neutralized by the condensation of positively charged guest molecules, we can expect neutral chain scaling for the torus size. However, it is not clear whether DNA is always neutralized upon collapse. In fact, recent experiments have suggested that in some conditions the interior charge of collapsed DNA is not completely neutralized, ${ }^{27,36}$ and thus there is some surviving net charge under some circumstances, which may lead to the unique collapsed morphology of DNA molecules. In such cases, we expect that our theory, though formulated for a weakly charged wormlike chain, could be used to understand the collapsing behavior of DNA molecules, at least, in a qualitative manner.

\section{ACKNOWLEDGMENTS}

The author wishes to thank K. Yoshikawa for valuable discussions. The present study was supported in part by a Grant-in-Aid for Scientific Research from the Ministry of Education, Culture, Sports, Science and Technology of Japan.

${ }^{1}$ P. G. de Gennes, Scaling Concepts in Polymer Physics (Cornell University Press, Ithaca, 1979).

${ }^{2}$ I. M. Lifshitz, A. Y. Grosberg, and A. R. Khokhlov, Rev. Mod. Phys. 50, 683 (1978).

${ }^{3}$ A. Y. Grosberg and A. R. Khokhlov, Statistical Physics of Macromolecules (American Institute of Physics, New York, 1994).

${ }^{4}$ C. B. Post and B. H. Zimm, Biopolymers 18, 1487 (1979).

${ }^{5}$ A. Y. Grosberg, Biofizika 24, 32 (1979).

${ }^{6}$ A. Y. Grosberg and A. R. Khokhlov, Adv. Polym. Sci. 41, 53 (1981).

${ }^{7}$ A. Y. Grosberg and A. V. Zhestkov, J. Biomol. Struct. Dyn. 3, 859 (1986).

${ }^{8}$ J. Ubbink and T. Odijk, Biophys. J. 68, 54 (1995).

${ }^{9}$ T. Odijk, J. Chem. Phys. 105, 1270 (1996).

${ }^{10}$ V. V. Vasilevskaya, A. R. Khokhkov, S. Kidoaki, and K. Yoshikawka, Biopolymers 41, 51 (1997).

${ }^{11}$ S. Y. Park, D. Harries, and W. M. Gelbart, Biophys. J. 75, 714 (1998).

${ }^{12}$ G. E. Plum, P. G. Arscott, and V. A. Bloomfield, Biopolymers 30, 631 (1990).

${ }^{13}$ V. A. Bloomfield, Biopolymers 31, 1471 (1991).

${ }^{14}$ V. A. Bloomfield, Curr. Opin. Struct. Biol. 6, 334 (1996).

${ }^{15}$ H. Noguchi, S. Saito, S. Kidoaki, and K. Yoshikawa, Chem. Phys. Lett. 261, 527 (1996).

${ }^{16}$ H. Noguchi and K. Yoshikawa, J. Chem. Phys. 109, 5070 (1998).

${ }^{17}$ V. A. Ivanov, W. Paul, and K. Binder, J. Chem. Phys. 109, 5659 (1998).

${ }^{18}$ Y. A. Kuznetsov and E. G. Timoshenko, J. Chem. Phys. 111, 3744 (1999).

${ }^{19}$ V. A. Ivanov, M. R. Stukan, V. V. Vasilevskaya, W. Paul, and K. Binder, Macromol. Theory Simul. 9, 488 (2000).

${ }^{20}$ M. R. Stukan, V. A. Ivanov, A. Y. Grosberg, W. Paul, and K. Binder, J. Chem. Phys. 118, 3392 (2003).

${ }^{21}$ T. Sakaue and K. Yoshikawa, J. Chem. Phys. 117, 6323 (2002).

${ }^{22}$ A. V. Dobrynin, M. Rubinstein, and S. P. Obukhov, Macromolecules 29, 2974 (1996).

${ }^{23}$ F. J. Solis and M. O. de la Cruz, Macromolecules 31, 5502 (1998).

${ }^{24}$ P. Chodanowski and S. Stoll, J. Chem. Phys. 111, 6069 (1999).

${ }^{25}$ A. V. Lyulin, B. Dunweg, O. V. Borisov, and A. A. Darinskii, Macromolecules 32, 3264 (1999).

${ }^{26}$ U. Micka and K. Kremer, Europhys. Lett. 49, 189 (2000).

${ }^{27}$ A. A. Zinchenko, V. G. Sergeyev, S. Murata, and K. Yoshikawa, J. Am. Chem. Soc. 125, 4414 (2003).

${ }^{28}$ F. Oosawa, Polyelectrolytes (Marcel Dekker, NewYork, 1971).

${ }^{29}$ G. S. Manning, Q. Rev. Biophys. 11, 179 (1978). 
${ }^{30}$ T. Odijk, J. Polym. Sci., Polym. Phys. Ed. 15, 477 (1977).

${ }^{31}$ J. Skolnick and M. Fixman, Macromolecules 10, 944 (1977).

${ }^{32}$ C. C. Conwell, I. D. Vilfan, and N. V. Hud, Proc. Natl. Acad. Sci. U.S.A. 100, 9296 (2003).

${ }^{33}$ A. Maritan, C. Micheletti, A. Trovato, and J. R. Banavar, Nature (London) 406, 287 (2000).
${ }^{34}$ W. M. Gelbart, R. F. Bruinsma, P. A. Pincus, and V. A. Parsegian, Phys. Today 53, 38 (2000).

${ }^{35}$ A. Y. Grosberg, T. T. Nquyen, and B. Shklovskii, Rev. Mod. Phys. 74, 329 (2002).

${ }^{36}$ Y. Yoshikawa, M. Suzuki, N. Chen, A. A. Zinchenko, S. Murata, T. Kanbe, T. Nakai, H. Oana, and K. Yoshikawa, Eur. J. Biochem. 270, 3101 (2003). 